Cumhuriyet Üniversitesi Fen Fakültesi Fen Bilimleri Dergisi (CFD), Cilt:36, No: 5 (2015) ISSN: $1300-1949$
Cumhuriyet University Faculty of Science

Science Journal (CSJ), Vol. 36, No: 5 (2015) ISSN: 1300-1949

http://dx.doi.org/10.17776/csj.56855

\title{
Boehler's Angle Estimations in Calcaneus Bone by Using Artificial Neural Networks
}

\author{
İlhan OTAĞ ${ }^{1}$, Serkan AKKOYUN ${ }^{1, *}$, Yaşar TAŞTEMUR ${ }^{2}$, Mehmet ÇİMEN² \\ ${ }^{l}$ Vocational School of Health, Cumhuriyet University, Sivas, Turkey \\ ${ }^{2}$ Faculty of Medicine, Department of Anatomy, Cumhuriyet University, Sivas, Turkey
}

Received: 02.03.2015; Accepted: 17.03.2015

\begin{abstract}
Boehler's angle has a great importance in diagnosis and treatment of calcaneus bones fractures. In this study, Boehler's angle was estimated by using artificial neural network method. This angle was obtained from 51 well-preserved calcaneus bones which was previously measured in anatomy laboratory at Cumhuriyet University. The data values for estimation belonging to these 51 different calcaneus bones are maximum anteroposterior length, maximum height, cuboidal facet height, body height and load arm length. By using this five different parameters, ANN estimation on Boehler's angle was performed. It is clearly seen from the results that the method is capable for the estimation.
\end{abstract}

Keywords: Boehler's angle, calcaneus bone, morphometry, artificial neural network

\section{INTRODUCTION}

Detailed knowledge about calcaneus anatomy can facilitate alternative treatment procedures [1]. Calcaneus is the biggest tarsal bone among all other tarsal bones. It makes up talocalcaneal/subtalar joint with talus [2]. Many foot diseases such as talocalcaneal artritis and coalition, intra-articular breaks, flatfoot relate to talus or calcaneus and other bones of the foot [3]. Talocalcaneal joint allows for eversion and inversion movements of the foot. Morphometric values of calcaneus are important for anatomy science, diagnosis procedure and treatment in orthopedic surgery, kinesiology, anthropology and forensic sciences [2]. Furthermore, calcaneus measurements are necessary for osteotomy [4]. Boehler's angle shows the strongest relationship for walking dynamic and produces clinical information for the researched issues. The main aim of treatment of calcaneal breaks reconstruction of Boehler's angle and restoration of calcaneus shape together with convenience of joint surface [5]. Boehler's angle is composed of the line combining posterior tuberosity of calcaneus and peak of posterior surface and the line between the peak of posterior surface and anterior process of calcaneus [6]. Calcaneus breaks are the most common tarsal breaks. Many breaks are obviously distinct. However, the breaks which are diagnosed hardly can only be diagnosed with low Boehler's angle [7]. Decrease of Boehler's angle is important for breaks. If the Boehler's angle belonging to the populations cannot be determined, many variations can be observed in angle measurement after operation [8].

\footnotetext{
*Corresponding author. Email: sakkoyun@ cumhuriyet.edu.tr
} 
In recent years, artificial neural network (ANN) method has been used in the bioengineering and biomechanics fields such as assessing parameters of gait symmetry [9], modeling leg and monofin movements [10], prediction of lower extremities' movement [11], bone fracture healing assessment [12] and classification of bone density [13]. As a mathematical model ANN mimics the human brain functionality. It consist of several processing units called neurons which are connected to each other by synaptic weights. Two main type of data is needed for the method, one is input and the other is output data. In this method, the desired outputs according to the given inputs are produced by appropriate weight modifications. In the present study, five different measurements belonging to the calcaneus bone were used as input parameters of the ANN. The aim is to obtain Boehler's angle according to the inputs. It was shown that the ANN method is useful for estimating Boehler's angles.

\section{MATERIAL AND METHODS}

\subsection{Material}

In our study, 51 well-preserved calcaneus bones in Laboratory of Anatomy Department at Cumhuriyet University were used. There was no information about the bones. Furthermore, due to the fact that there is no any significant relation of Boehler's angle to age, gender or side of body, the data was not grouped according to these differences and considered as a whole. In calcaneus analyses, 5 distances were measured and called as parameters. Sixth measurement was Boehler's angle (Fig. 1). These parameters are maximum anteroposterior length $(M L)$, maximum height $(M H)$, cuboidal facet height $(C F H)$, body height $(B H)$, load arm length $(L A L)$ and Boehler's angle $(B A)$. These lines are; 1 -superior magrin of posterior facet to superior magrin on anterior process 2-superior magrin of posterior facet to superior magrin of tuberosity. A sliding caliper with sensitivity of $0.1 \mathrm{~mm}$ was used in linear measurements and a goniometer was used in measurement of $B A$.

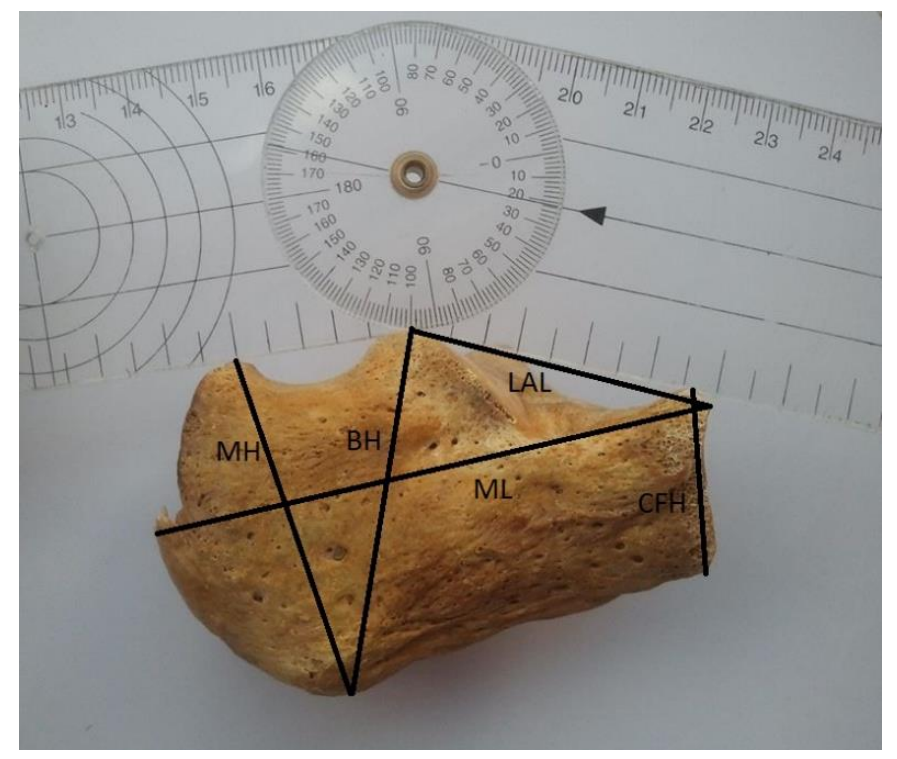

Figure 1. Measurement points used in calcaneus bone; ML; maximum anteroposterior length, MH; maximum height, $\mathrm{CFH}$; cuboidal facet height, BH; body height and LAL; load arm length. 


\subsection{Method of the analysis}

In order to estimate Boehler's angle, the measured calcaneus bone values (5 parameters) and Boehler's angle mentioned in Section 2.1 were used. Artificial neural network (ANN) method was used as a tool for this estimation. The main task of the ANN is to give outputs in consequence of the computation of the inputs. ANN is a mathematical model that mimics the human brain and used in many fields for solving complex problems. It is composed of several processing units intitled neurons which have adaptive synaptic weights [14]. ANN is also efficient tools for pattern recognition. The ANN consists of three main layers: input, hidden and output layers. Each layer has own neurons named as input, hidden and output neurons. The neurons in each layers are connected to the others in the next layers of own. The input neurons received the data from environment and transmit via the connections. Then corresponding output data is given by the output neurons. In this study, one input layer with five neurons, two hidden layer with six $(h=4)$ neurons in each and one output layer with one neuron (5-4-4-1) ANN topology was used for precisely prediction of Boehler's angle (Fig. 2). The hidden layer and neuron numbers were taken as optimal ones after several trials. The total number of adjustable weights (w) without bias was 40 according to the formula given by

$w=p x h_{1}+h_{1} x h_{2}+h_{2} x r$

where $p$ and $r$ are the neuron numbers in input and output layers, $h_{1}$ and $h_{2}$ are hidden neuron numbers in the first and second hidden layers, respectively.

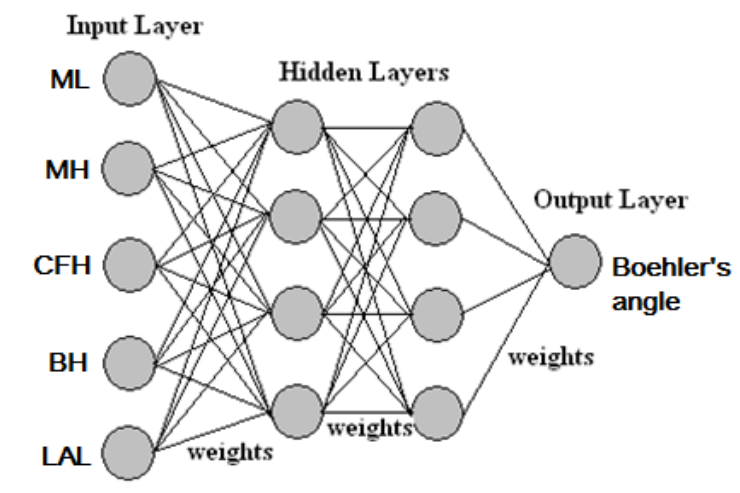

Figure 2. The used ANN architecture (5-4-4-1). The neurons in each layer are connected to the next layer neurons by adaptive synaptic weights.

Hidden layer is needed for approximation any nonlinear function. The activation function of neurons in this layer can be any well-behaved nonlinear function. In this work we chose sigmoidlike (hyperbolic tangent) activation function for hidden layers whose formula given by 
$\tanh =\frac{\left(e^{x}-e^{-x}\right)}{\left(e^{x}+e^{-x}\right)}$

NeuroSolutions v6.02 [15] was used for calculations as an ANN software. All data (total 305) were divided into two parts. One of this is for the training stage and the rest is for the test stage. For the training stage, the algorithm was a back-propagation algorithm with LevenbergMarquardt [16, 17]. By appropriate modifications, ANN modifies its all weights until an acceptable error level between estimated and desired outputs is reached. The difference between these different outputs was measured by mean square error (MSE) given by

$M S E=\frac{\left[\sum_{k=1}^{r} \sum_{i=1}^{N}\left(y_{k i}-f_{k i}\right)^{2}\right]}{N}$

where $N$ is the number of training or test data, $y_{k \mathrm{i}}$ and $f_{k i}$ are the desired output and ANN output, respectively. After training of the ANN with final weights, the network performance is tested over an unseen data in the training stage. If the estimations of the test data are sufficiently good, ANN is considered to have learned the functional relationship between input and output data.

\section{RESULTS AND DISCUSSION}

The ANN method which is successful in the case of highly non-linear data as in this present study was applied on the data that was assigned for the training stage. The input parameters of the ANN were $M L, M H, C F H, B H$ and $L A L$ (see Fig. 1). In Table 1, it was shown the range of the parameters used. As can be seen in the table that the $B A$ values were lied between $20^{\circ}$ and $40^{\circ}$.

Table 1. Range of the parameters used as inputs and output.

\begin{tabular}{|l|l|}
\hline Parameter & Range \\
\hline$M L$ & $62.5-87.5$ \\
\hline$M H$ & $38.1-54.7$ \\
\hline$C F H$ & $17.3-33.5$ \\
\hline$B H$ & $44.0-60.7$ \\
\hline$L A L$ & $42.8-54.6$ \\
\hline$B A$ & $20.0-40.0$ \\
\hline
\end{tabular}

The desired output according to the given input parameters was measured values of the $B A$ $\left(B A_{\text {meas }}\right)$. After training stage, the final weights were obtained for the study. First by using these final weights, the trained ANN was tested over the training data. According to the results which are clearly seen in the Fig. 3 that the constructed ANN was successful for estimation of $B A$. The deviations of ANN results from measured values were concentrated near the zero. The maximum 
deviation is $1^{\circ}$. The MSE value between $B A_{\text {meas }}$ and $B A_{A N N}$ (estimated by the ANN) for the training stage was obtained as 0.146 . The maximum epoch number was 1000 in the training. The error minimization process during this stage was shown in Fig. 4. The final training mean square error value was approximately $6 \times 10^{-4}$.

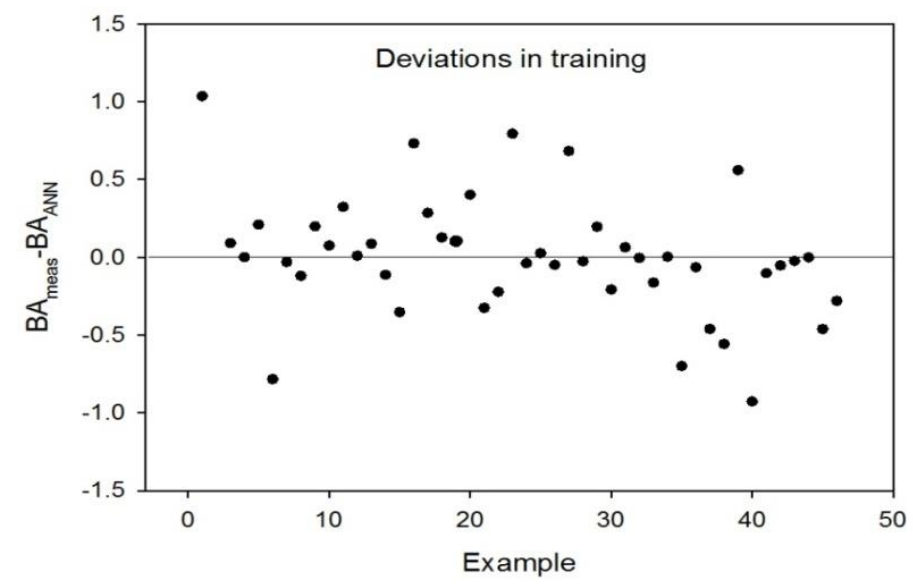

Figure 3. Deviation of ANN estimation from measured values.

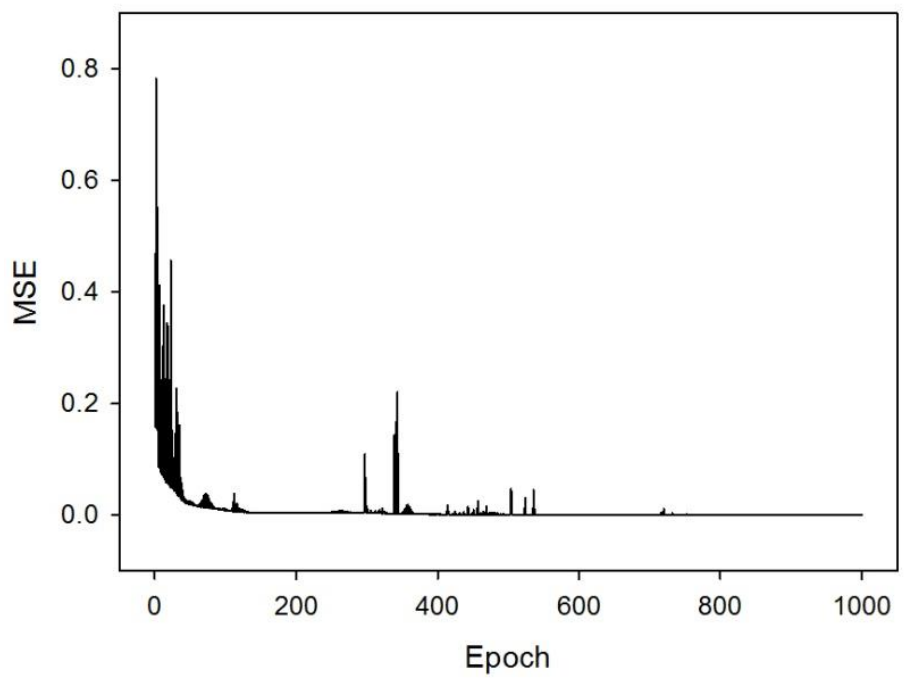

Figure 4. Error minimization in training process with epoch number 1000.

It was concluded from the training results that the constructed ANN is ready for the test stage. It is not sufficient to see the success of the ANN only on training data. The constructed ANN with its final weights have to be tested over the test data which were never used in the training stage. The results of the test stage show the generalization ability of the ANN. If the estimations of the ANN is good enough over the test data, one can confidently says that the ANN generalized the data. The test of the ANN is performed on the test data. The results from test stage were given in Table 2 ranked according to the increasing deviation values. Clearly seen in the table, the ANN 
estimations were very similar to the highly nonlinear measured values of $B A$ with maximum deviation of $-1.24^{\circ}$. The MSE value for the training stage was obtained as 0.767 .

Table 2. ANN predictions for Boehler's angle $\left(\mathrm{BA}_{\mathrm{ANN}}\right)$ and deviations from measurements $\left(\mathrm{BA}_{\mathrm{meas}}\right)$. This data was not used in the training stage.

\begin{tabular}{|c|c|c|c|c|c|c|c|}
\hline MAXL & MAXH & CFH & BH & LAL & BAmeas & BA ANN $_{\text {Deviation }}$ & \\
\hline & & & & & & & \\
\hline & & & & & & & \\
\hline & & & & & & & \\
\hline & & & & & & & \\
\hline & & & & & & & \\
\hline 72.8 & 44.2 & 28.2 & 47.1 & 46.3 & 24 & 24.01 & -0.01 \\
\hline 79 & 48 & 21.5 & 54.7 & 45.2 & 30 & 29.97 & +0.03 \\
\hline 74.3 & 49.3 & 23.3 & 56.6 & 49 & 34 & 34.76 & -0.76 \\
\hline 85.3 & 47.6 & 24 & 59.5 & 50.7 & 29 & 29.91 & -0.91 \\
\hline 82.5 & 46.1 & 25.6 & 50.2 & 51.2 & 32 & 33.24 & -1.24 \\
\hline
\end{tabular}

It was seen in the previous works [18] that the studies on $B A$ is performed over different populations. Also it is noticed that an angle ranges belonging to the different populations were determined. In the present study of ANN, the $B A$ was estimated with maximum deviation of $1.24^{\circ}$. These results also remain the identified $B A$ range. By using the ANN method, the results can be useful for restructuring operations of calcaneus fractures. Thus, measuring the five parameters about calcaneus bone helps calculations of $B A$ whose correct determination is very important for the body structure and position. In addition, in the case of absence of one or more parameters of these five, it was seen that $B A$ can also be identified by little more deviations. But the results of this type of work were not presented here to stand on the main purpose of the study.

\section{CONCLUSIONS}

This work suggest an alternative method, ANN, for obtaining $B A$ value which is crucial in diagnosis and treatment of calcaneus fractures. The ANN method used different five measurements belonging to the calcaneus bone and gives the appropriate $B A$. It was clearly shown that the results obtained from ANN are in good agreement with the measured data. Particularly, our results indicate that one should take predictive power of ANN into account. The method has several advantage such as speed in calculations and no need any relationship between input and output data. Also in the case of absence some of the measurements belonging to the calcaneus bone, the method has still high predictive power on determining the $B A$.

\section{REFERENCES}

[1] Kwak Y.H., Park K.B., Park H.W. Kim H.W., Use of allograft in skeletally immature patients for calcaneal neck lengthening osteotomy, Yonsei Med. J., 2008, 49(1), 79-83. 
[2] Kidd R.S., Oxnard C.E., Patterns of morphological discrimination in selected human tarsal elements, Am. J. Phys. Antropol., 2002, 117, 169-181.

[3] Zwipp H., Rammelt S., Subtalare arthrodese mit calcaneus-osteotomie, Orthopade, 2006, 35(4), 387-406.

[4] Doğan A., Albayrak M., Akman Y.E., Zorer G., The results of calcaneal lengthening osteotomy for the treatment of flexible pes planovalgus and evalution of alignment of the foot, Acta Orthop. Tramatol. Turc., 2006, 40(5), 356-366.

[5] Rosenbaum D., Lübke B., Bauer G., Claes L., Long-term effects hindfoot fractures evaluated by means of plantar pressure analyses, Clin. Biomech., 1995, 10(7), 345-351.

[6] Boehler L., Diagnosis, patology and treament of fractures of the as calcis, J. Bone Joint Surg., 1931, 13, 75-89.

[7] Nicholson D.A., O'keeffe D., Driscoll P.A., The foot, BMS, 1993, 307, 997-1001.

[8] Işiklar Z.U., Bilen F.E., Fractures of calcaneus, J.Totbid, 2006, 5(1-2), 44-52.

[9] Michalski R., Wit A., Gajewski J., Use of artificial neural networks for assessing parameters of gait symmetry, Acta Bioengineering and Biomechanics, 2011, 13 (4), 65-70.

[10] Rejman M., The elements of modeling leg and monofin movements using a neural network, Acta Bioengineering and Biomechanics, 2006, 8 (1), 53-61.

[11] Kutilek P., Farkasova B., Prediction of lower extremities' movement by angle-angle diagrams and neural networks, Acta Bioengineering and Biomechanics, 2011, 13 (2), 5765.

[12] Kaufman J.J. et al., A neural network approach for bone fracture healing assessment, IEEE Eng. Med. Biol. Mag. 1990, 9(3), 23-30.

[13] Özerdem M.S., Akpolat V., Yapay Sinir Ağları ile Kemik Yoğunluğunun Sınıflandırılması, IEEE 15. Sinyal İşleme ve İletişim Uygulamaları Kurultayı, 2007, Eskişehir.

[14] Haykin S., Neural networks: a comprehensive foundation, 2nd ed, Prentice-Hall, New Jersey, 1999.

[15] Levenberg K.A., Method for the Solution of Certain Non-Linear Problems in Least Squares, Quart. Appl. Math., 1944, 2, 164-168.

[16] Marquardt D., An Algorithm for Least-Squares Estimation of Nonlinear Parameters, SIAM J. Appl. Math., 1963, 11, 431-441.

[17] Neurosolutions, http://www.neurosolutions.com/.

[18] Igbigni P.S., Mutesasira A.N., Calcaneal angle in Ugandans, Clinical Anatomy, 2003, 16, 328-330. 